\title{
Differential effects of communication on operant behavior in children
}

\author{
JOSEPH S. EDWARDS, DIANE DeA. EDWARDS, and JOANNE LUCAS \\ University of Missouri at Kansas City, CB Annex, Room 100, Kansas City, Missouri 64110
}

\begin{abstract}
Performance on a free-operant differentiation device which required subjects to press one of two panels for candy and money was used to study the effects of nonvisual communication and listening to a story in seven children (normal and exceptional) and one adult. Differential responding to the panel that produced reinforcement was acquired by all subjects in the first session. Communication by a relevant adult (mother) decreased reinforced and nonreinforced responding and increased talking. Listening to a story decreased both reinforced and nonreinforced responding but did not increase talking. Communication had no effect on any of the behaviors of an autistic child and increased the speech of a retarded child the least of the subjects who talked. The effect of crying in one child was also found to decrease responding.
\end{abstract}

In 1962, Lindsley first described a closed-circuit television system for styding the ongoing process of dyadic communication (looking, listening, talking). Video and audio access between members of the dyad was continuously made possible via conjugate reinforcement servos. For each member to listen and see the televised image of his partner, foot or hand presses on a simple switch at a preset rate of response was required. Response rate continuously altered the listening and looking levels. High response rates maintained the video image and audio channels at full intensity. Momentary reductions in rate resulted in immediate reductions of the visual image and audio intensity. Using this system, communication in psychotherapy (therapist and psychotic), between patients (psychotic and psychotic) (Lindsley, 1968, Note 1), in nurse training (nursing supervisor and supervisee) (Nathan, Marland, \& Lindlsey, 1965), and during psychiatric admission interviews (Nathan, Schneller, \& Lindsley, 1964) has been studied.

The method was demonstrated to be sensitive to: (1) type of partner (therapist, psychotic, friend, nursing supervisor), (2) type of speech, (3) content of speech, (4) psychotic state of the individual, and (5) menstrual cycle in a nursing supervisor. Communication was studied as a behavioral process. Three behavioral components were directly recorded under a variety of dyad and experimental conditions. All three behaviors (looking, listening, talking) were found to be sensitive indicators of variables intrinsic and extrinsic to the communication. Important to the present research was the finding that responses to look at and listen to one's partner are independent behaviors in both normal and psychotic persons (Lindsley, 1968; Nathan et al., 1964; Lindsley, Note 1).

The present study is the first of a series designed to evaluate dyad nonvisual communication as an independent variable on reinforced and nonreinforced manual responding and talking. The nonvisual dimension was selected since much of human communication exists in this form and because data from the previous research suggested the independence of these modalities in communication. This research sought to determine how communication, as a social behavior, operates on specific behaviors of young children.

Three behaviors: reinforced panel pressing, nonreinforced panel pressing (manual behaviors), and talking were studied. Since the previous research had shown that social communication conditions within dyads as well as external conditions could alter the reinforcement value of looking, listening, and talking both within and across sessions, we sought to determine the function of communication on behaviors in a simple, ongoing operant task.

\section{METHOD}

Subjects

Six children ( 3.5 years to 8 years) and one adult ( 24 years) participated. One child (C.A., 7 years), was diagnosed as mentally retarded (WISC IQ 60) and one child as autistic (6 years). The children were recruited from mothers in the undergraduate and graduate psychology program.

\footnotetext{
Apparatus

Experimental sessions were conducted in a sound attenuated cubicle. The cubicle contained a chair, appropriate to the child's height, facing a sloping console mounted on the rear wall. The console contained two Plexiglas response panels $(4 \times 6$ in.) and a reinforcement delivery tray. Presses on either panel $(30 \mathrm{~g}$, approximate force, through a distance of $.5 \mathrm{~mm}$ ) closed microswitches which operated digital counters, cumulative recorders, and other appropriate standard relay circuitry. Talking was recorded through a voice-operated relay onto counters and a cumulative recorder. A Grason-Stadler E1105 timer was inserted between the voice-operated relay and a pulse former to limit the maximum vocal output rate to 120 responses per minute. This duration filter was added to minimize "overcounts" due to changes in the amplitude of the subject's speech and other extraneous sounds. The response panels were covered with foam rubber on the back surface so that neither maximum rates or banging the panels would produce voice counts.
} 


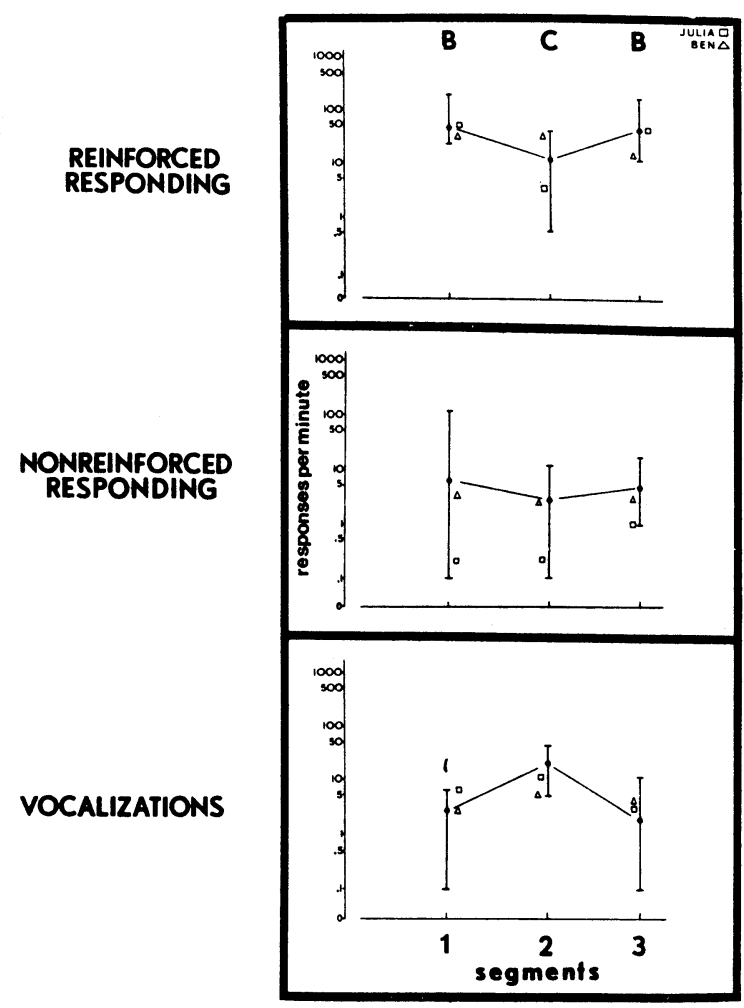

Figure 1. Overall performance of all subjects during baseline (B) and communication conditions (C).

\section{Procedure}

All subjects participated in two to four 30-min sessions spaced approximately 3 days apart. Each session consisted of three conditions: reinforcement without communication, reinforcement with communication, and reinforcement without communication. Presses on the right panel facing the subjects produced M\&M's candy and a penny on a variable-interval (VI) 30 -sec schedule of reinforcement. Nickels were used with the adult. Presses on the left panel had no consequence.

Subjects wore cushioned headphones throughout each session. Within session changes from no communication to communication conditions were unannounced to the subjects. The communication partner for each child was his or her mother. The partner for the adult was her major professor.

In three children, communication to mother and listening to mother read a story were compared. Calibration and testing of the audio system before each session showed that panel pressing did not discharge the voice-operated relay and that pressing the panels did not interfere with clear audio reception by the subjects.

Records of reinforced panel presses, nonreinforced panel presses, and talking of each subject were continuously recorded throughout all sessions. Communication and noncommunication conditions lasted approximately $10 \mathrm{~min}$ each or until stable responding was visually apparent on the cumulative recorders.

Since the task involved differential responding, the criterion for analyzing baseline differential responding (presses on the reinforced panel/presses on the nonreinforced panel) was a $75 \%$ rate difference in $75 \%$ of the baseline segments across sessions.

At the beginning of each session, the subjects were comfortably seated before the console and instructed that, "These panels have something to do with earning candy and money. When you earn a piece of candy or money, it will fall into this tray." The experimenter pointed to the tray and with great enthusiam stated, "Look what I found (two M\&M's candies and a penny). Now, I want you to wear these earphones all the time. Are you ready? When I close the door you may start. When the session is over, your mother and I will come to get you."

\section{RESULTS}

Overall, the results of the experiment showed that: (1) All subjects showed evidence of differential responding to the panel that produced reinforcement (median rate difference of $83 \%$ ), (2) communication in all but the autistic boy reduced reinforced and nonreinforced manual response rates (median decreases of $80 \%$ and $84 \%$, respectively) and increased talking (median rate increase of 94\%) above baseline levels, (3) listening to a story being read produced similar decreases in behavior as did communication, but talking by the children did not increase, (4) the autistic boy was the only subject whose behavior remained unchanged by communication, and (5) crying in one child following communication was also accompanied by a deceleration in all behavioral responses. (6) the retarded girl talked at the lowest rate of the children who communicated.

Figure 1 presents median response rates and ranges for the three behaviors during baseline, communication, and baseline conditions. In the intial and final baseline conditions, it is evident that reinforced panel pressing occurs at higher rates than nonreinforced pressing and that talking is infrequent. Communication decreased responses to both panels similarly in spite of wide differences in the absolute rate of response. Talking increased dramatically only during communication conditions.

The two exceptional children, Julie (Down's Syndrome) and Ben (autistic) behaved differentially. Julie's behavior on the reinforced and nonreinforced panels clearly shows evidence of differential responding and sensitivity to the communication variable. Although Ben differentially responded to the two panels, no effect is apparent in any of his behaviors during the communication condition. In spite of the differential responding and the reduction in panel pressing during communication, Julie talked the least of those children who communicated.

Figure 2 presents sample cumulative records of three subjects that show within session changes in response rate to communication and listening to a story. In the first panel, Diane's performance during baseline (Segments 1 and 3), is characterized by a high stable rate on the reinforced panel, a near zero rate on the nonreinforced panel, and little talking. During communication (Segment 2), reinforced panel pressing was reduced by approximately $50 \%$ of the baseline rate. Responding during this condition is characterized by an overall rate reduction rather than a change in the pattern of responding. Christy's performance during baseline (Segments 1, 3, and 5), listening to a story being read (Segment 2), and cummunication (Segment 4) is 
presented in the second panel. This session was selected because it shows atypical performance by Christy, and it clearly shows the effect of an emotional condition (crying) on operant behavior. Initial session performance during baseline (Segment 1) is characterized by high rate responding on both panels with a reduction of reinforced and nonreinforced panel pressing toward the end of that condition. This reduction in responding is paralleled by a gradual acceleration in vocalizations (which went unanswered). Analysis of the tape recording of this session revealed that Christy's vocalizations consisted of complaints about participating in the experiment on this day and requests for communication with her mother. Listening to a story being read (Segment 2) reduced all behaviors to near zero rate levels. Reintroduction of baseline conditions (Segment 3) generated little reinforced responding with a low, erratic rate of crying throughout this period. Communication conditions were instituted in Segment 4 . This condition is also characterized by near zero rates on the reinforced and nonreinforced panels. Vocalization consisted of mixed cries and attempts to communicate. A brief reinstatement of baseline conditions (Segment 5), initially accelerated crying (in the absence of panel pressing). This acceleration was abruptly followed by zero rates during the remaining portion of the session.

Julie's behavior during baseline (Segments 1 and 3) and communication (Segment 2) are presented in the last panel. Baseline conditions are characterized by stable rates of reinforced panel pressing, zero rate of nonreinforced panel pressing, and some vocalizations. Communication reduced reinforced panel pressing and only slightly increased vocalizations.

\section{DISCUSSION}

The data from this research show a strong potential for utilizing the method for long- and short-term investigations of the social reinforcement derived from a partner's speech, the type of speech engaged in, and clinically relevant data on the extent of the responsiveness of exceptional children to communication. In every child except the one labeled autistic, and in the one adult, the freely programmed opportunity to communicate, accelerated talking and decelerated materially reinforced and nonreinforced responding. Mechanically, it was possible to both talk, listen, and panel press simultaneously. Analysis of within session conversation and postsession interviews revealed statements by the children such as, "Mama, stop talking to me, I can't work!" Some mothers replied on several occasions, "You can work while I talk to you," and the frequent reply was, "No, I can't ... why are you doing it?"

It is important to note that differentiation of the appropriate response was acquired rapidly and that differential responding was maintained with all children under most conditions. Whether communication would continue to function as a declerator of materially reinforced behavior can only be determined by long-term investigations. In addition, methodological changes in the type of communication and making the communication opportunity dependent on a specific response would provide a direct means of determining the ongoing reinforcement value of communication relevant to material reinforcement (Lucas \& Edwards). The use of more complicated behaviors would also permit a more refined analysis of the effect of communication.

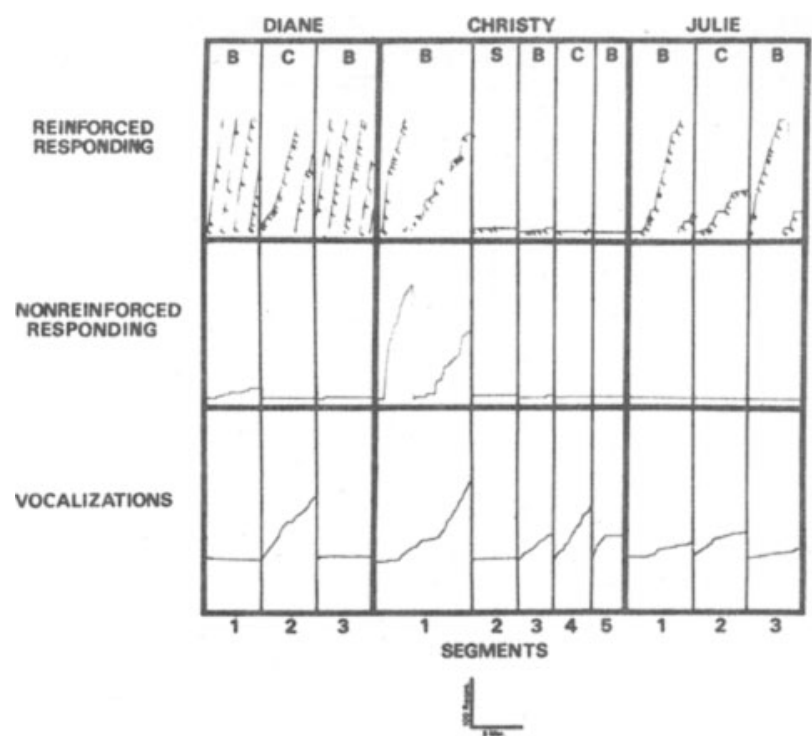

Figure 2. Within session performance of three subjects shows typical effects of communication in a normal (Diane) and a Down's Syndrome child (Julie) and disruption in behavior during an atypical crying episode (Christy).

Of all the children who talked, Julie's rate (Down's Syndrome) was the lowest. This effect occurred in conjunction with a reduction in reinforced and nonreinforced panel pressing. This finding is of interest since Julie's vocabulary of spoken words was estimated to be approximately 30 . Julia's spoken vocabulary was the smallest of any of the other children. Ben's (autistic) vocabulary was estimated by his parents and a professional as being in excess of 500 words. Future research efforts in this area will have to carefully select and program conditions to differentiate children who have the language to communicate vs. those children who listen but have a more limited communication repertoire as well as those children who have the capability but do not respond to the opportunity. Description and remedial programs would obviously be different for the different types of children.

The results from the atypical crying session with Christy support data on other intrasubject variables obtained earlier by Lindsley and his associates $(1964,1965$, Note 1) with psychotic patients and normal adults. Precise specifications as how such emotional conditions such as crying effect selected human behaviors is worthy of investigation in its own right and especially in regard to how such states affect individual and social behaviors.

\section{REFERENCE NOTE}

1. Lindsley, O. R. Direct behavioral analy sis of psy chotherapy sessions by conjugately programmed closed-circuit television. Paper read at American Psychological Association, St. Louis, Missouri, September 1962.

\section{REFERENCES}

Lindsley, O. R. Direct behavioral analysis of psychotherapy sessions by conjugately programmed closed-circuit television. Psychotherapy, Theory, Research and Practice, 1969, 6, 71-81.

Nathan, P. E., Schneller, P., \& Lindsley, O. R. Direct measurement of communication during psychiatric admission interviews. Behavioral Research Therapy, 1964, 2, 49-57.

Nathan, P. E., Marland, J., \& Lindsley, O. R. Receptive communication in psychiatric nurse supervision. Journal of Counseling Psy chology, 12, No. 3, 1965. 